\title{
IoT and AWS based Precision Agriculture in Ayurvedic Betel Leaf Cultivation
}

\author{
${ }^{1}$ Sanjay H.M, ${ }^{2}$ Manogna.D.Raj \\ ${ }^{1}$ Associate Professor, ${ }^{2}$ M.Tech, PES College of Engineering, Mandya, India, \\ ${ }^{1}$ sanjaypesce@gmail.com, ${ }^{2}$ manognadevraj@gmail.com
}

Abstract The main aim of this paper is to keep an eye on the growing betel leaf inside the greenhouse using Internet of Things (IoT). Maintaining the growth of the betel leaf helps in the quality and quantity of the production throughout the year. Since betel leaf can be grown all over many years one can maintain the data of the surrounding temperature, soil moisture, its climbing stems are also observed and these data are recorded and stored in the Amazon Web Server (AWS). For this we use Wireless Sensor Networks (WSN) such as temperature sensor, soil moisture sensor, accelerometer sensor, relay, water pump. These sensors help in detecting any changes inside the greenhouse and those data are stored in AWS and can be retrieved whenever necessary. GSM Module allows mobile phone to receive message through GPRS.

Keywords---Internet of things (IoT), Renesas Microcontroller, Wireless sensor network (WSN), Amazon Web Services (AWS). General Packet Radio Service (GPRS), Liquid Crystal Display (LCD).

\section{INTRODUCTION}

The demanding interest for crop creation and quality has basically dilated in the usage of wonderful nursery or greenhouse. The dilated people requests for large live of yield creation. We are able to develop the harvests which require some specific natural conditions within the nursery. This task portrays the set up and execution of remote detector systems for nursery condition perceptive. The Wireless Sensor Network (WSN) is one among the foremost essential innovations without delay. Without delay can exhibit a top level view of the IoT wonders even as its applications on nursery. IoT may be a dream of a world wherever most articles square measure associated; transmission refreshes concerning their presentation therefore the people who use them to try to things all the additional with wisdom. The essential plan driving the IoT is that for all intents and functions every physical factor without delay likewise becomes a computer that's related to the online. Without delay detector can discover the elemental parameters of nature. The detected qualities are shown on a digital display show. The sensors square measure related to the microcontroller. the straightforward sensors associated through Associate in Nursing ADC. The message is send to the noncommissioned variety through GSM. The engine is ON naturally if the dirt wetness is a smaller amount. Microcontroller may be a computer gift in an exceedingly solitary coordinated circuit that is committed to perform one assignment and execute one express application. It contains a processor center, memory and programmable information or yield peripherals. The microcontroller used here is that the R5F100LE that may be a RL78 family smart microcontroller. GSM electronic equipment may be specific electronic equipment that acknowledges a SIM card and works over a membership to versatile activity, a lot of constant as a mobile phone. AT commands square measure used to regulate the GSM modems. Detector may be a device whose object is to spot changes in its condition, and subsequently provides a relating yield. The actual changes may well be within the lightweight, heat, movement, dampness, pressure, or any of a rare variety of alternative ecological marvels. A .Net internet application is introduced with EC2 example of Amazon internet server, since once the warning message got, and constant checking qualities are shown on website and chart is created with Pentaho ETL instrument and tableau programming therefore values with diagram is effectively to look at varieties in natural conditions.

\section{RELATED WORK}

A literature review could be a body of text that aims to review the essential points of current information and method approaches on a selected topic. For complete study of the system there's a requirement to travel through every and each technical fact of the connected material exhaustive. Conferred below is that the survey of associated technologies and outline of connected work exhausted the past. The survey done by the author's focus is on a lot of energy-efficient IoT as Associate in Nursing enabler of varied applications together with agriculture. Specially, it aims to present a large set of models dealing with the 
agriculture. Special attention and determination is paid for the growth of the plant and Amazon Web Server (AWS). Nowadays, to reduce the manual work in agricultural field many smart farming technologies are induced by using IoT sensors which has the ability to provide information within the agricultural fields. By implementing technologies like IoT the major factor is to improve the yields of the efficient crops. It monitors temperature and humidity using certain sensors and also captures images and sent to the farmer's mobile phone via Wi-Fi. IoT based monitoring system in smart agriculture [9]. In this paper they have proposed a system which can collect information inside the greenhouse and the collected information helps to predict the changes in environment and helps in controlling the climatic conditions. They use certain sensors to predict the variations inside the greenhouse and monitors the crop to increase the yields and it also has certain advantages such as high testing precision, accuracy and clear data management. Computerized Environmental Control in Greenhouses [10]. Farmers faced many problems when all the agricultural field works was manually like farming resources optimization, decision making ability and land monitoring. All these problems are solved by the use of WSN in the agriculture. Using WSN provides the farmers to take real time decisions at the right time. By the use of WSN and the IoT technologies one can come up with the good and healthy crops throughout the year. Wireless sensor network in precision agriculture application [18]. Was proposed based on the small and medium sized greenhouse environment which is based on GSM technology and Radio Frequency(RF).It combines the remote communication technology with the GSM networks and allows the system to implement the small and medium sized greenhouse environment monitoring. It has achieved the real time monitoring of greenhouse environment by showing its stability, reliability and is capable of achieving in real time. The design and implementation of the greenhouse monitoring system based on GSM and RF technologies [13].

\section{III.EXISTING SYSTEM}

Nurseries in India are being conveyed in the high-elevation locales where the below zero temperature up to $-40^{\circ} \mathrm{C}$ makes any sort of ranch practically outlandish and in dry areas where conditions for plant development are antagonistic. The current arrangements essentially are:

Laboring system: Laboring system setup contains graphic scrutiny of plant growth, manual plant irrigation, turning the temperature controllers $\mathrm{ON}$ and $\mathrm{OFF}$, manual chemical spraying and pesticides. It takes time, is susceptible to human error and so less correct and unreliable.

Complete mechanized system: Incomplete mechanized system set-up could be a grouping of manual superintendence and partial mechanization and is analogous in most respects to manual set-up however reduces the work needed to irrigate the set-up.

Completely mechanized system: This can be a complicated set-up that is well furnished to retort to most of the climate changes going down within the greenhouse. It works on a response system that helps it respond effectively to the external stimulation. although this set-up overcomes the issues caused by human errors, it's not absolutely automatic and expensive. Errors, it isn't fully automated and costly.

\section{IV.PROPOSED SYSTEM}

From the present downside, it will be seen that the prevailing systems are lean to handle the issues of greenhouse observation and management. To resolve these issues, we tend to propose greenhouse observation and management exploitation IoT and AWS. It consists principally of the sensors, the controllers, observation half, causing and receiving a message. Temperature device, soil moisture device, measuring system device are underneath the observation section. These sensors are accustomed sense the varied environmental conditions and also the values detected by the sensors can seem on the LCD digital display alphanumeric display. All the sensors are connected to the Renesas Microcontroller (R5F100LE) that may be a controller. The ADC are connected via analog sensors. The water pump is then connected to microcontroller through a relay. If any changes in the surface within the environmental set-up, an alert message is shipped to the registered mobile number through GPRS. Succeeding half is that the causing and receiving of the message. There's a GSM Module within the causing half that may send the data to the Amazon Web Server (AWS). The receiver half is an Amazon Web Server with an application that plays a predefined message supported the text warning received from module GSM. Cube suit++ provides the benefit to increase the potential of the programs.

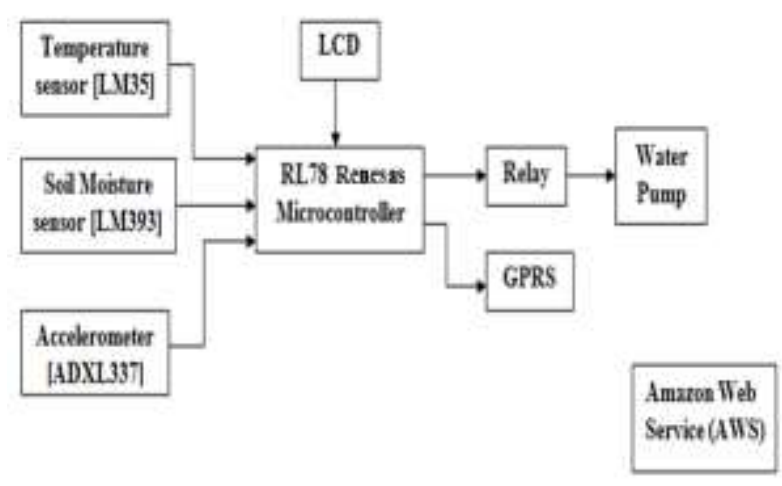

Figure 4.1 Block Diagram of System Architecture

Many embedded systems have considerably completely different styles per their functions and utilities. The microcontroller settled at the center of the diagram forms 
the management unit of the whole system. Embedded at intervals the microcontroller may be a program that helps the microcontroller to require action supported the inputs provided by the output of the sensors. Here Amazon Web Service (AWS) and Internet of Things (IoT) square measure used. Wireless detector networks like measuring instrument, soil wet detector and temperature detector, whereas the whole unit alongside Amazon server, fulfills the thought of IoT. Accelerometers square measure mounted close to the betel pepper leaf. Any changes in position of the vines, produces and output voltage against gravity. The output of those measuring instrument sensors that is accelerometer sensor square measure given to the ADC unit of the microcontroller. Supported the program embedded at intervals the microcontroller the voltages generated by the measuring instrument square measure displayed on the LCD. Soil Moisture sensors square measure placed within the soil at completely different places at intervals the farm. Output of those sensors, square measure given to the ADC unit of the microcontroller. supported the program embedded at intervals the microcontroller the voltages generated by the soil sensors square measure are displayed on the LCD. Temperature sensors square measure located in a completely different places at intervals of the farm. Result of those sensors, square measure given to the ADC unit of the microcontroller. Supported the program embedded at intervals the microcontroller the voltages generated by the temperature square measure displayed on the LCD. All the sensors connected to the microcontroller alongside GPRS, forms one entire node. Output of such nodes, placed at completely different places, at intervals the farm, is shipped to the Amazon server. Since betel pepper leaf cultivation is fully grown for many years the knowledge of each year is maintained within the Amazon Web Server (AWS). The storage of data in AWS helps to maintain the data for several years and storing of data also helps in the retrieval of data whenever required.

Advantages of the projected system are:

1. Any plant kind will be grown throughout the year thanks to automatic dominant of setting.

2. Reduces manual work.

3. Prevents the danger of failure by human errors at bound environmental conditions.

4. Decreases the labor price by having a greenhouse.

5. AWS keeps the record of the whole conditions of the plant throughout the year.

6. If any changes surface within the greenhouse, message is shipped to the owner.

\section{RESULTS}

These constructions were designed for the management setting and check the parameters of the Piper betel leaf. Supported Effective greenhouse environmental management the output of the machine-driven approach to the world manual approach area unit adopted. The encryption is predicated on absolutely automatic management control. Microcontroller device Modules, this method obtains correct result from any of the input parameter. The setting in greenhouse will be terribly helpful for human intervention at that point. Soil wetness device and therefore the wetness device area unit connected via ADC as a result of their analog sensors. The sensors have the ability to sense the various parameters and therefore the current output are going to be showed within the liquid crystal display and that they can send the messages to the owner via GPRS. AWS stores the data sent by the sensors from the start of the crop conditions throughout the year. Data is stored for every 2 minutes in AWS.

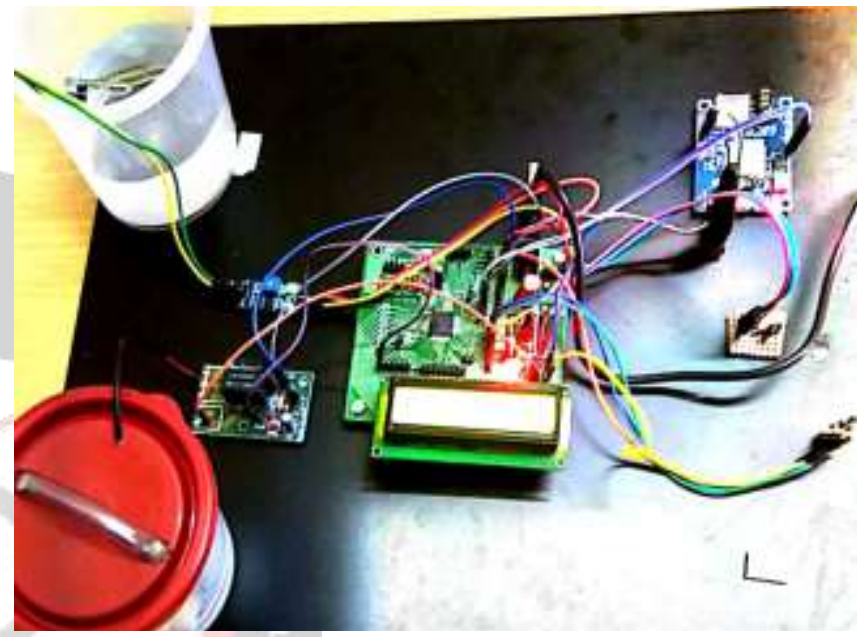

Figure 5.1 Overall setup of Betel Leaf Cultivation

The above figure 5.1 represents the overall setup of the Betel Leaf Cultivation. All the sensors like temperature sensor, soil moisture sensor, accelerometer sensor, water pump and relay are connected to the Renesas microcontroller. Renesas microcontroller controls the overall setup. LCD is connected to the Renesas board which displays the temperature value, soil moisture value and also if any changes in the growth of the plant is displayed on the LCD display.

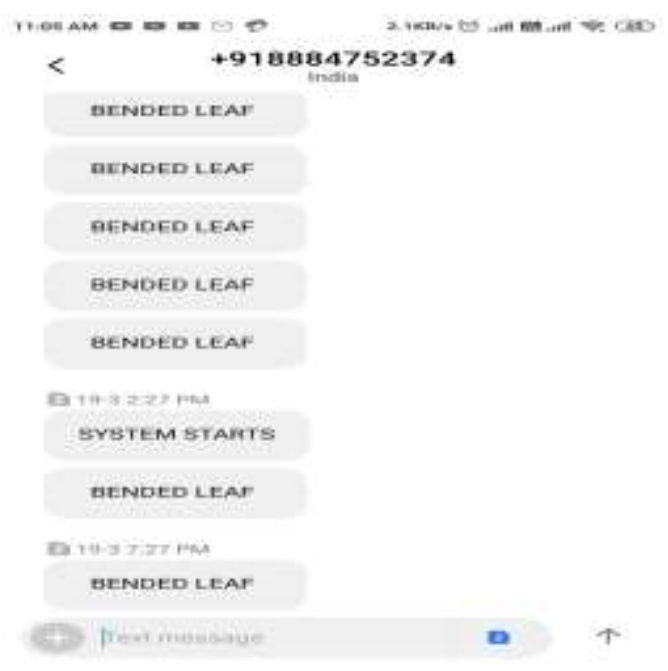

Figure 5.2 Message displayed on mobile phone via GPRS 
The above figure 5.2 is the mobile display message. The message is displayed on the registered mobile number via General Packet Radio Service (GPRS). It is related to the accelerometer sensor which means accelerometer sensor is used to sense the change in the position to growing plant. If any change with respect to the change in the position of the plant sends the bended leaf message to the mobile number through GPRS.

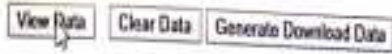

\begin{tabular}{|c|c|c|c|c|c|c|}
\hline Timet & Date & Tenpernture & Mristun & $x$ & $\mathbf{Y}$ & Pusp \\
\hline 1105.15 & $2030-03.19$ & 30 & 167 & 169 & 130 & 1 \\
\hline 110503 & 20.203 .19 & 00 & $\omega s$ & WO & owo & 0 \\
\hline 110158 & 2030.03 .19 & 00 & 00 & $\cos$ & 601. & 0 \\
\hline 105827 & 2010.03 .19 & 99 & 195 & 165 & 158 & 1 \\
\hline 105643 & $2020-0=19$ & 30 & 34 & 146 & 160 & 1 \\
\hline 105404 & 202005.19 & 99 & 497 & 164 & 127 & $\theta$ \\
\hline 165138 & 2020.02 .19 & 00 & 600 & $\cos$ & $\cos$ & 0 \\
\hline $20+850$ & $20009-18$ & 12 & 202 & 090 & 80 & 1 \\
\hline $20+734$ & $200-04-18$ & 09 & 00 & 000 & 000 & 0 \\
\hline
\end{tabular}

Figure 5.3 Data stored in AWS

The above figure 5.3 represents the data stored in Amazon Web Server (AWS). Since betel leaf cultivation can be carried out above 5-6 years continuously, each and every day data must be preserved because it helps in the future to view the data of the past cultivation which also helps in the increase in the quality and quantity of the production. To achieve this, we use AWS to store the data of the cultivation.

\section{CONCLUSION}

This paper describes the look of Associate in Greenhouse IoT based mostly greenhouse watching system for the cultivation of Piper betel leaf vine. In recent times, agricultural comes area unit on the increase, in distinctive forms, even in urban areas. Technological advances create the agricultural sector increase high within which IoT is creating here. IoT can dramatically amendment however we have a tendency to live our daily lives, and also the data regarding to it is kept. Wireless sensors and GSM will produce the updates, these watching system percepts completely different parameters among the greenhouse. The system developed will prove profitable because it can optimize the greenhouse resources. The entire module is affordable, low-power, straightforward to access everyone, reduces manual work. This paper may be an elementary plan of greenhouse analysis, however there's still far more to be explored technologically.

\section{FUTURE ENHANCEMENT}

In this paper we've got enclosed the detectors like temperature sensor, soil moisture detector, accelerometer sensor; relay and water pump that helps in maintaining the environmental conditions within the greenhouse. Any changes within the greenhouse area unit sophisticated via GPRS. However, the disease caused for the pepper vine leaf or the betel stem or on the betel root and also the type of disease occurring on the leaf or stem or root and also the curative measures to cure the disease are often increased within the future work. The identification of the disease on the betel leaf can be made by image processing in the future work.

\section{REFERENCES}

[1]G.Sandhi, F.Buemi, M. Massa, M. Zucchini, "Visually guided operations in green-houses", IEEE International Workshop on Intelligent Robots and Systems, 1990

[2]K.Rangan and T.Vigneswaran, "An Embedded Systems Approach to Monitor Green House", 978-1-424491827/10/\$26.00 @2010 IEEE

[3]Wei Ai and Cifa Chen, "Green House Environment Monitor Technology Implementation Based on Android Mobile Platform", 978-1-4577-0536-6/11/\$26.00 @2011 IEEE

[4]Akshay C., NitinKarnwal, Abhfeeth K.A., Rohan Khandelwal, Tapas Govindraju, Ezhilarasi D and Sujan Y., "Wireless sensing and control for precision Greenhouse management", 978-1-4673-2248-5/12/\$31.00 (C2012 IEEE

[5]AjiHanggoro, Mahesa Adhitya Putra, Rizki Reynaldo, RiriFitri Sari, "Green House Monitoring and Controlling Using Android Mobile Application", 978-1-4673-57852/13/\$31.00 @2013 IEEE

[6]S.Thenmozhi, M.M.Dhivya, R.Sudharsan and K.Nirmalakumari, "Greenhouse Management Using Embedded System and Zigbee Technology", IJAREEIE, Vol. 3, Issue 2, February 2014

[7]M.K. Gayatri, J.Jayasakthi and Dr.G.S. Anandha Mala, "Providing Smart Agricultural Solutions to Farmers for better yielding using Iot",2015 IEEE International Conference on Technological Innovations in ICT for Agriculture and Rural Development(TIAR 2015)

[8]P.S.Asolkar, U.S.Bhadade, "An Effective Method of Controlling the Greenhouse and Crop Monitoring Using GSM”, 2015 International Conference on computing communication control and automation.

[9]S.P.Pratibha, Anupama Hongal, M.P.Jyothi, "IoT base monitioning system in smart agriculture", 2017 
International conference onrecent advances in electronics and communications.

[10]G.J.Timmerman, P.G.H.Kamp, "Computerized Environmental Control in Greenhouses", PTC the Netherlands, pp. 15-124, 2003.

[11]J.W. Mastalerz, "The greenhouse enironment: the effect of environmental factors on the growth and development of flower crops", New York:Wiley, 1977.

[12]. K.Rangan, T. Vigneswaran, "An Embedded systems approach to monitor green house", Recent Advances in Space Technology Services and Climate Change (RSTSCC), 2010.

[13]Xiaoyan, Z. Xiangyang, D. Chen, C. Zhaohui, S. Shangming, and Z. Zhaohui, "The design and implementation of the greenhouse monitoring system based on GSM and RF technologies," in Computational Problemsolving (ICCP), 2013 International Conference on, Oct 2013, pp. 32-35.

[14]Nikesh Gondchawar, R. S. Kawitkar, "IoT based Smart Agriculture", International Journal of Advanced Research in Computer and Communication Engineering, vol. 5, no. 6, pp. 2278-1021, June 2016.

[15] P. Rajalakshmi, S. Devi Mahalakshmi, "IoT Based Crop-Field Monitoring and Irrigation Automation" in 10th International conference on Intelligent systems and control (ISCO) 7-8 Jan 2016, published in IEEE Xplore, Nov 2016.

[16]Tanmay Baranwal, Nitika Pushpendra Kumar Pateriya, "Development of IoT based Smart Security and Monitoring Devices for Agriculture" in 6th International Conference Cloud System and Big Data Engineering, IEEE, pp. 978-14673-8203-8/16, 2016.

[17]. Nelson Sales, Artur Arsenio, "Wireless Sensor and Actuator System for Smart Irrigation on the Cloud" in 9781-5090-0366-2/15 2nd World forum on Internet of Things (WF-IoT) Dec 2015, published in IEEE Xplore, jan 2016.

[18]. Mohamed Rawidean Mohd Kassim, Ibrahim Mat, Ahmad Nizar Harun," Wireless Sensor Network in Precision Agriculture Application”, pp. 978-1.

[19]Mohamed Rawidean Mohd Kassim, Ibrahim Mat, Ahmad Nizar Harun, "Wireless Sensor Network in Precision agriculture application" in International conference on computer Information and telecommunication systems (CITS), published in IEEE Xplore, July 2014.

[20]Ankit Yadav," Design and implementation of smart agriculture using embedded system", 2016 International journal of advanced trends in computer science and engineering.
[21]M.S.Mekala, Viswanathan Perumal, "ASurvey: Smart agriculture IoT with cloud computing”, 2017 International conference on microelectronic devices, circuits and system (ICMDCS).

[22]Laxmi Shabadi, Hemavati B, "Design and Implementation of IoT based smart security and monitoring for connected smart farming", 2018 International journal of computer applications. 